\title{
Inverse Compensation for Hysteresis in Magnetostrictive Transducers
}

\author{
Ralph C. Smith \\ Center for Research in Scientific Computation \\ Department of Mathematics \\ North Carolina State University \\ Raleigh, NC, 27695 \\ rsmith@eos.ncsu.edu
}

\begin{abstract}
This paper addresses the development of inverse compensation techniques for a class of ferromagnetic transducers including magnetostrictive actuators. If unaccommodated, the hysteresis and nonlinear dynamics can produce severe loss of control authority and potential instabilities when the actuators are incorporated in control design. In this work, hysteresis is modeled through the domain wall theory originally proposed by Jiles and Atherton. This model is based on the quantification of the energy required to translate domain walls pinned at inclusions in the material with the magnetization at a given field level specified through the solution of an ordinary differential equation. A complementary differential equation is then employed to compute the inverse which can be used to compensate for hysteresis and nonlinear dynamics in control design. The performance of the inverse compensator and its employment in LQR control design are illustrated through numerical examples.
\end{abstract}

Keywords: Inverse compensation, hysteresis, magnetostrictive materials 


\section{Introduction}

Increased demands on control transducers in combination with novel material designs have led to the development of a class of ferromagnetic transducers, including those based on magnetostrictive materials, which deliver high level strains and forces but often exhibit significant hysteresis and nonlinear dynamics. While magnetostrictive transducers can be utilized in linear regimes by maintaining low input levels, control requirements often dictate that they be driven at the high drive levels where hysteresis and nonlinearities are inherent to the actuator dynamics. Furthermore, the advantages of these transducers over traditional actuators is typically realized at high drive levels. Hence it is necessary to design control methods which accommodate the observed actuator hysteresis and nonlinearities.

While numerous mechanisms can produce hysteresis and nonlinearities in magnetostrictive actuators, a primary source is attributed to ferromagnetic domain mechanisms. This produces sigmoid hysteresis curves of the type illustrated in Figure 1 for the magnetostrictive material Terfenol-D and is the form of hysteresis that we consider here.

To illustrate the issues pertinent to control design using high performance actuators, we consider a plant with nonlinear input $u$ provided by the actuator as indicated in Figure 2 . The input to the actuator is denoted by $v$ while the dynamics of the actuator are represented by $N(\cdot)$. We note that the map $N(\cdot)$ is typically both nonlinear and a multivalued function of the input level due to the hysteresis. It is, however, a well-defined function of $v$. Finally, we let $u_{p}$ denote the input specified to obtain the control objective in the absence of the nonlinearities and hysteresis $N(\cdot)$.

For systems exhibiting even mild hysteresis, linear control methods are typically ineffective due to incurred phase shifts and unmodeled energy loss in the hysteresis loop. To address this, we consider the construction of exact and approximate inverses for the actuator nonlinearities and hysteresis. This entails the computation of a map $N^{-1}(\cdot)$ or approximate map $\widehat{N}^{-1}(\cdot)$ such that $u_{p}=N^{-1}(v) \approx \widehat{N}^{-1}(v)$. For linear control implementation, $u_{p}$ is filtered by $N^{-1}(\cdot)$

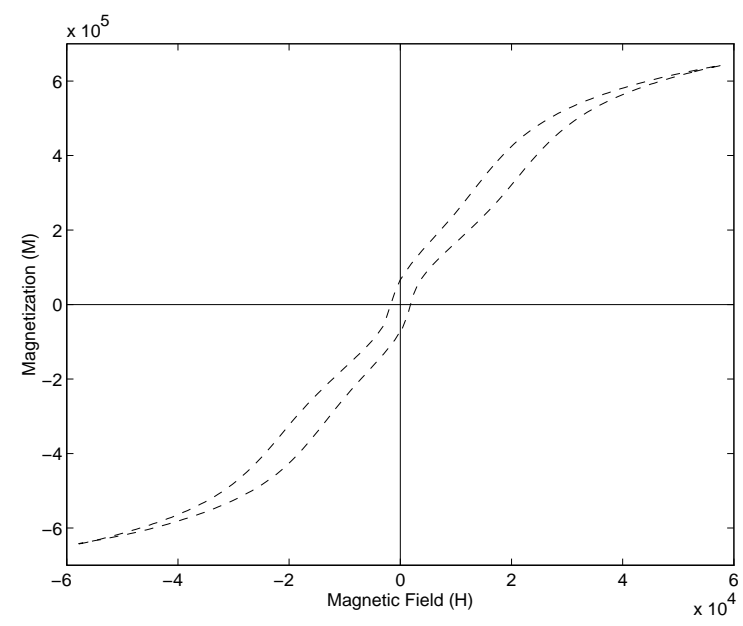

Figure 1. Relationship between the magnetic field $H$ and magnetization $M$ for Terfenol-D (Experimental data collected at Iowa State University). 


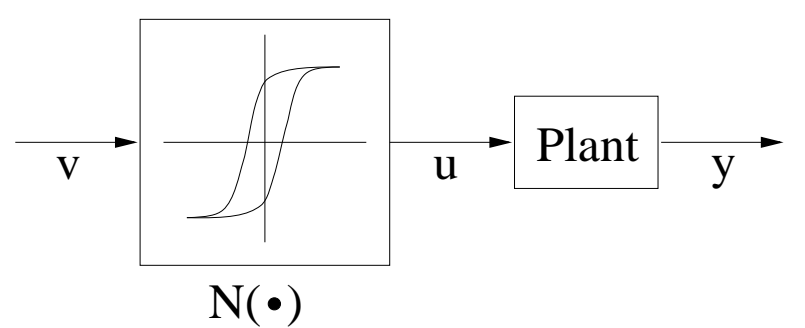

(a)

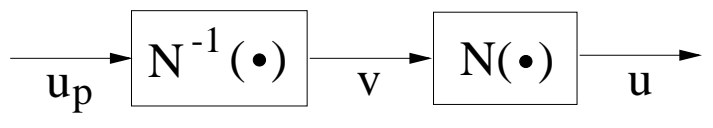

(b)

Figure 2. (a) Plant with input actuator hysteresis $N(\cdot)$; (b) Inverse compensator $N^{-1}(\cdot)$ for the hysteresis.

before input to the filter. In this manner, one compensates for the nonlinearity and the final signal $u=N\left(N^{-1}\left(u_{p}\right)\right)$ is that prescribed by the control law. This process is illustrated in Figure 2.

We emphasize that the process of inverse compensation for nonlinearities is well known and the reader is referred to [1] for a comprehensive discussion in the context of general actuator nonlinearities and hysteresis. The models employed in [1] to characterize the hysteresis $N(\cdot)$ and inverse $N^{-1}(\cdot)$ are based on piecewise linear Preisach approximations and hence are phenomenological in nature. The contribution of this paper lies in the construction of a hysteresis model and inverse for magnetostrictive actuators which is based upon energy principles. This yields a compensation filter which is easily constructed and can be readily updated to accommodate changing operating conditions.

The model for the actuator hysteresis process $N(\cdot)$ is based upon the theory of Jiles and Atherton $[2,3]$. This theory is based upon the tenant that ferromagnetic hysteresis is primarily due to the energy required to bend and translate domain walls pinned at inclusions in the material. By formulating the energy of domain wall characteristics in terms of bulk material properties, Jiles and Atherton obtained a model which requires only five parameters. The construction of the model is further enhanced by the physical nature of the parameters and the fact that certain parameters can be directly specified from measured data. This model has subsequently been extended to magnetostrictive transducers $[4,5]$ and it is in this setting that we consider the development of an inverse $N^{-1}(\cdot)$. We note that while the model and inverse compensator are illustrated in the context of LQR feedback control, the techniques are equally applicable when designing feedforward or adaptive control systems. Again, the reader is referred to [1] for a comprehensive discussion of adaptive control design for systems exhibiting input hysteresis once an exact or approximate inverse has been determined.

Section 2 provides a summary of the hysteresis model and the inverse model is developed in Section 3. The use of the inverse compensation scheme for optimal control design in a structural system is illustrated in Section 4. For that development, we consider magnetostrictive transducers employed as control actuators for attenuating vibrations in a thin beam. The hysteretic actuator inputs $N(\cdot)$ are incorporated in a thin beam model and the dynamics are approximated to obtain a finite dimensional control system. Finally, numerical examples are provided to illustrate the performance of an LQR control with the inverse $N^{-1}(\cdot)$ used to compensate for the actuator hysteresis and nonlinearities. 


\section{Hysteresis Model}

The model described here is based on the tenant that hysteresis in ferromagnetic materials is due to the impeded movement of domain walls which are pinned at inclusions or defects in the material. In such materials, changes in magnetization are attributed to the nucleation and growth of domains through domain wall movement (note that domains denote regions in which moments are highly aligned while domain walls are the transition regions between domains). For materials which are free from inclusions, domain wall movement is unimpeded and the magnetization is anhysteretic (hysteresis-free). This situation is idealized, however, since defects are unavoidable (e.g., carbons in steel) and in many cases, incorporated in the material to achieve the desired stoichiometry (e.g., Dysprosium in Terfenol-D). These defects or inclusions provide pinning sites for domain walls due to the reduction in energy which occurs when the domain intersects the pinning site. For low magnetic field variations about some equilibrium value, the walls remain pinned and the magnetization is reversible. This motion becomes irreversible at higher field levels due to wall intersections with remote pinning sites. The model quantifies hysteresis through the characterization of the anhysteretic magnetization $M_{a n}$, the reversible magnetization $M_{r e v}$ and the irreversible magnetization $M_{i r r}$.

The anhysteretic magnetization at a point in the material is dependent on the effective magnetic field, the saturation magnetization and the thermal energy of the sample. Under the assumption of constant stress $\sigma_{0}$, the effective field can be expressed as

$$
H_{e f f}=H+\alpha M
$$

where $H$ denotes the external field input to the transducer, $M$ denotes the magnetization and the parameter $\alpha$ quantifies the effects of interdomain coupling and magnetoelastic domain interactions. Two commonly employed models for the anhysteretic are the Langevin expression

$$
M_{a n}=M_{s}\left[\operatorname{coth}\left(\frac{H_{e f f}}{a}\right)-\left(\frac{a}{H_{e f f}}\right)\right]
$$

and the Ising spin relation

$$
M_{a n}=M_{s} \tanh \left(\frac{H_{e f f}}{a}\right) .
$$

Both models are derived using arguments from Boltzmann statistics with the differences dependent upon the assumptions concerning possible domain orientations. Taylor expansions of the hyperbolic cotangent and tangent functions reveals that the models agree through third

order terms with the Ising spin model producing steeper slopes $\frac{d M_{a n}}{d H}$ due to the magnitude of the higher order terms. The constant $a$ is given by $a=\frac{\mathcal{N} k_{B} T}{\mu_{0} M_{s}}$, where $k_{B}$ is Boltzmann's constant, $k_{B} T$ represents the Boltzmann thermal energy, $\mu_{0}$ is the free space permeability, and $\mathcal{N}$ is the average domain density. The saturation magnetization $M_{s}$ can often be specified from measured data or a priori knowledge about the material. The parameter $a$, on the other hand, must be estimated through either a least squares fit to data or adaptive parameter estimation techniques since $\mathcal{N}$ is unknown. Due to its dependence on the temperature $T$, this parameter may vary during operation, in which case, adaptive estimation and control techniques may be preferable. 
Hysteresis losses are incorporated through the quantification of energy required to bend and translate domain walls. As developed in [2], an irreversible component to the magnetization $M_{i r r}$ can be computed through the consideration of energy dissipation due to pinning and unpinning of domain walls at inclusions in the material. This yields the expression

$$
\frac{d M_{i r r}}{d H}=\frac{M_{a n}-M_{i r r}}{k \delta-\alpha\left(M_{a n}-M_{i r r}\right)}
$$

for the differential susceptibility where $k=\frac{\langle p\rangle\left\langle\varepsilon_{\pi}\right\rangle}{2 m \mu_{0}(1-c)}$, and $\langle p\rangle$ is the average density of pinning sites, $\left\langle\varepsilon_{\pi}\right\rangle$ is the average energy for $180^{\circ}$ walls, $c$ is a reversibility coefficient, and $m$ is the magnetic moment of a typical domain. Through its definition, $k$ provides a measure for the average energy required to break a pinning site. The parameter $\delta$ is defined to have the value +1 when $d H>0$ and -1 when $d H<0$ to guarantee that pinning always opposes changes in magnetization. In applications, $\delta$ can be directly determined from the magnetic field data while $k$ is identified for the specific transducer and operating conditions.

We note that in certain regimes near saturation, the expression (3) can produce nonphysical results. Specifically, when $d H$ is reversed near saturation, the expression (3) can produce negative values of the differential susceptibility which are not observed in experiments. Following the strategy detailed in [3], we consider the initial changes in magnetization to be due to the reversible relaxation of bulged domain walls when $d H$ is reversed and enforce $\frac{d M_{i r r}}{d H}=0$. This produces the more physically realistic expression

$$
\frac{d M_{i r r}}{d H}=\widetilde{\delta} \frac{M_{a n}-M_{i r r}}{k \delta-\alpha\left(M_{a n}-M_{i r r}\right)}
$$

where

$$
\widetilde{\delta}=\left\{\begin{array}{l}
1,\left\{d H>0 \text { and } M>M_{a n}\right\} \text { or }\left\{d H<0 \text { and } M<M_{a n}\right\} \\
0, \text { otherwise } .
\end{array}\right.
$$

The reversible magnetization quantifies the degree to which domain walls bulge before attaining the energy necessary to break the pinning sites. As derived in [2], to first approximation, the reversible magnetization is given by

$$
M_{r e v}=c\left(M_{a n}-M_{i r r}\right) \text {. }
$$

The reversibility coefficient $c$ can be estimated from the ratio of the initial and anhysteretic differential susceptibilities [3] or through a least squares fit to data.

The total magnetization is then given by

$$
M=M_{r e v}+M_{i r r}
$$

with $M_{i r r}$ and $M_{r e v}$ defined by (4) and (5) and the anhysteretic magnetization given by (1) or (2). To provide a relation which facilitates inversion, it is advantageous to express the output magnetization as a function of the input field. When the Ising spin model is used to characterize the anhysteretic magnetization, the expressions (2), (4) and (5) can be 
consolidated to yield

$$
\begin{aligned}
\frac{d M}{d H} & =(1-c) \frac{d M_{i r r}}{d H}+c \frac{d M_{a n}}{d H} \\
& =\widetilde{\delta}(1-c) \frac{M_{a n}-M_{i r r}}{k \delta-\alpha\left(M_{a n}-M_{i r r}\right)}+c \frac{d M_{a n}}{d H} \\
& =\frac{\widetilde{\delta}\left(M_{a n}-M\right)}{k \delta-\frac{\alpha}{1-c}\left(M_{a n}-M\right)}+c \frac{d M_{a n}}{d H} \\
& =\frac{\tilde{\delta}\left[M_{s} \tanh \left(\frac{H+\alpha M}{a}\right)-M\right]}{k \delta-\tilde{\alpha}\left[M_{s} \tanh \left(\frac{H+\alpha M}{a}\right)-M\right]}+\frac{c M_{s}}{a} \operatorname{sech}^{2}\left(\frac{H+\alpha M}{a}\right)\left(1+\alpha \frac{d M}{d H}\right)
\end{aligned}
$$

where $\tilde{\alpha}=\frac{\alpha}{1-c}$. The magnetization at a given field level is then specified by the solution to the differential equation

$$
\begin{aligned}
& \frac{d M}{d H}=\mathcal{F}(H, M) \\
& M\left(H_{0}\right)=M_{0}
\end{aligned}
$$

where

$$
\begin{aligned}
& \mathcal{F}(H, M)=\frac{1}{1-\frac{c M_{s} \alpha}{a} \operatorname{sech}^{2}\left(\frac{H+\alpha M}{a}\right)} \cdot\left\{\frac{\widetilde{\delta}\left[M_{s} \tanh \left(\frac{H+\alpha M}{a}\right)-M\right]}{k \delta-\tilde{\alpha}\left[M_{s} \tanh \left(\frac{H+\alpha M}{a}\right)-M\right]}\right. \\
& \left.+\frac{c M_{s}}{a} \operatorname{sech}^{2}\left(\frac{H+\alpha M}{a}\right)\right\} .
\end{aligned}
$$

If one employs the Langevin expression (1) rather than the Ising spin relation (2) for the anhysteretic, the function $\mathcal{F}$ is given by

$$
\begin{array}{r}
\mathcal{F}(H, M)=\frac{1}{1+\frac{c M_{s} \alpha}{a}\left[\operatorname{csch}^{2}\left(\frac{H+\alpha M}{a}\right)-\left(\frac{a}{H+\alpha M}\right)^{2}\right]} \cdot\left\{\frac{\widetilde{\delta}\left[M_{s} \mathcal{L}\left(\frac{H+\alpha M}{a}\right)-M\right]}{k \delta-\tilde{\alpha}\left[M_{s} \mathcal{L}\left(\frac{H+\alpha M}{a}\right)-M\right]}\right. \\
\left.\quad-\frac{c M_{s}}{a}\left[\operatorname{csch}^{2}\left(\frac{H+\alpha M}{a}\right)-\left(\frac{a}{H+\alpha M}\right)^{2}\right]\right\}
\end{array}
$$

where the Langevin function is defined by

$$
\mathcal{L}(z) \equiv \operatorname{coth}(z)-\frac{1}{z}
$$

The relation (7) with $\mathcal{F}$ given by (8) or (9) is then employed when quantifying the actuator hysteresis $N(\cdot)$. In both cases, the parameters $\delta$ and $\widetilde{\delta}$ can be computed directly from the measured field data or the computed values of the magnetization and anhysteretic magnetization. The saturation magnetization $M_{s}$ can often be determined directly from the measured data or a priori knowledge of the material behavior. Hence only the parameters $a, \alpha, c$ and 
$k$ must be determined through a least squares fit to data or adaptive parameter estimation techniques.

Finally, we note that the time-dependent dynamics of the magnetization can be specified through the chain rule

$$
\frac{d M}{d t}=\mathcal{F}(H, M) \frac{d H}{d t} .
$$

This relation should be employed only for quasistatic processes, however, since this model neglects the elastic properties of the material as well as time-dependent loss mechanisms.

\section{Inverse Hysteresis Model}

The hysteresis model in the form (7) is amenable to inversion through consideration of a complementary differential equation. For actuators in which the anhysteretic is modeled by the Ising spin model, the inverse is specified through solution of the differential equation

$$
\begin{aligned}
& \frac{d M^{-1}}{d H}=\frac{1}{\mathcal{F}\left(M^{-1}, H\right)} \\
& M^{-1}\left(H_{0}\right)=M_{0}^{-1}
\end{aligned}
$$

with $\mathcal{F}$ defined by (8). Similarly, employment of (9) provides the inverse when the Langevin model is used to characterize the anhysteretic magnetization.

It can be readily verified that the inverse defined through (10) satisfies

$$
M\left(M^{-1}(H)\right)=M^{-1}(M(H))=H
$$

for an input field $H$. In the control nomenclature of Section 1, the definition (10) can be used to provide the exact inverse $N^{-1}(\cdot)$ if the parameters $M_{s}, \alpha, a, c$ and $k$ are known exactly. For the examples in this paper, it is assumed that suitable parameters have been obtained through a least squares fit to data and the exact inverse is employed. Similarly it provides an approximate inverse $\widehat{N}^{-1}(\cdot)$ which is suitable for adaptive parameter estimation and control methods if the parameters are unknown or slowly change due to changing operating conditions.

\section{Example 1.}

In control applications, one typically employs the computed inverse as a filter to compensate for the physical hysteresis and nonlinearities produced by the actuator. Hence one considers the operation

$$
u=N\left(N^{-1}\left(u_{p}\right)\right)
$$

where $u_{p}$ is the specified control in the absence of actuator hysteresis and nonlinearities. This example illustrates the inversion process with the hysteresis specified by $N=M$.

The model (7) and inverse (10) were constructed with the parameters $M_{s}=0.280 \mathrm{C} / \mathrm{m}^{2}$, $a=4.2 \times 10^{5} \mathrm{C} / \mathrm{m}^{2}, k=3.7 \times 10^{5} \mathrm{C} / \mathrm{m}^{2}, \alpha=1.5 \times 10^{6}, c=0.65$. A $1 \mathrm{~Hz}$ piecewise linear signal $u_{p}$ having a maximum magnitude of $0.25 \mathrm{~V} / \mathrm{m}$ was employed as input. Such quasistatic signals are commonly employed in material characterization experiments. A first-order forward 
difference method (Euler's method) was used to numerically integrate the differential equations (7) and (10). Due to the high sample rates in typical experiments, the method provides sufficient accuracy, efficiency and stability for both numerical and real time experimental implementation.

The input signal $u_{p}$ and output signal $u=M\left(M^{-1}\left(u_{p}\right)\right)$ are plotted in Figure 3 while the inverse function $v=M^{-1}\left(u_{p}\right)$ is plotted in Figure 4. A comparison of $u_{p}$ and $M\left(M^{-1}\left(u_{p}\right)\right)$ illustrates the qualitative accuracy of the inversion process while numerical computations indicate that the absolute errors introduced in the process have magnitude less than $10^{-14}$. While such accuracy obviously cannot be expected when the finite dimensional inverse $N^{-1}(\cdot)$ is employed in an infinite dimensional process $N(\cdot)$, the maintenance of these accuracy levels under approximation does illustrate the stability of the method.

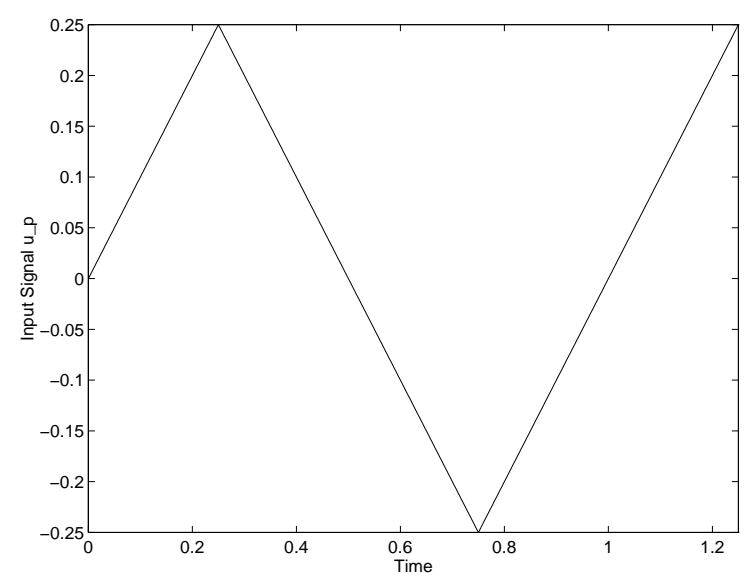

(a)

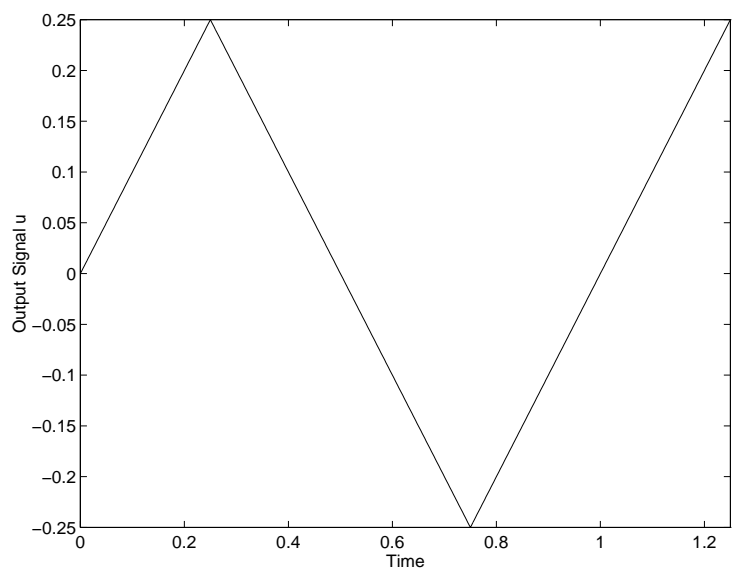

(b)

Figure 3. (a) Signal $u_{p}(t)$ input to the inverse compensator; (b) Signal $u(t)=M\left(M^{-1}\left(u_{p}(t)\right)\right)$ applied to the plant.

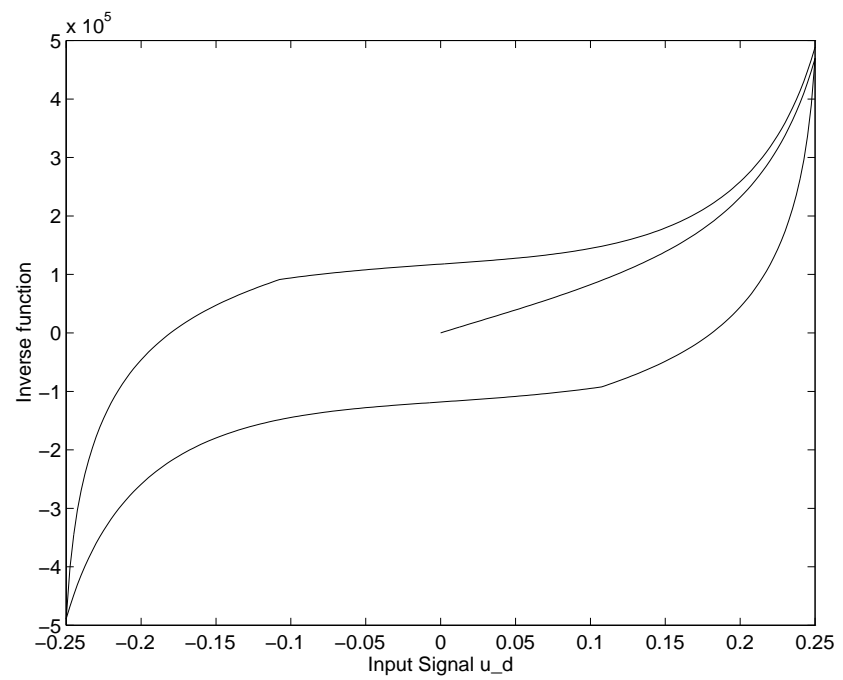

Figure 4. Inverse function $M^{-1}\left(u_{p}\right)$. 


\section{Structural Application and Control Example}

To illustrate the incorporation of the actuator hysteresis model in a structural model and indicate the utility of the inverse compensator in linear control design, we consider a cantilevered thin beam with end-mounted magnetostrictive actuators as depicted in Figure 5. Spatially uniform forces drive the beam while diametrically out-of-phase currents to the actuators generate bending moments which attenuate transverse beam vibrations. This setup has been employed in experiments designed to ascertain capabilities and properties of Terfenol-D transducers [6] and it provides a prototype for illustrating the attributes of the inverse compensator. For further details concerning the construction of the actuator, the reader is referred to $[6]$.

For this example, we will employ an LQR feedback control law. However, the inverse compensator is equally effective for linear feedforward or adaptive control methods as dictated by the application.

For modeling purposes, the beam is assumed to have length $\ell$, width $b$, and thickness $h$. The density, Young's modulus, Kelvin-Voigt damping coefficient and air damping coefficient for the beam are denoted by $\rho_{b}, E_{b}, c_{D_{b}}$ and $\gamma$, respectively. The cross-sectional area of the Terfenol rod is denoted by $A_{m a g}$ while the Young's modulus and damping coefficient for the Terfenol rod are denoted by $E^{H}$ and $c_{D}^{H}$. The length and width of the connecting bar are denoted by $\ell_{r}$ and $b_{r}$, respectively, while the bar density is given by $\rho_{r}$. Finally, the transverse beam displacement is given by $w$ while $g(t, x)$ denotes an exogenous surface force to the beam.

Moment and force balancing yields the strong form of the Euler-Bernoulli equations

$$
\begin{aligned}
& \rho(x) \frac{\partial^{2} w}{\partial t^{2}}(t, x)+\gamma \frac{\partial w}{\partial t}(t, x)+\frac{\partial^{2} \mathcal{M}_{\text {int }}}{\partial x^{2}}(t, x)=g(t, x)+\frac{\partial^{2} \mathcal{M}_{\text {mag }}}{\partial x^{2}}(t, x), \quad \begin{array}{c}
0<x<\ell \\
t>0
\end{array} \\
& \left.\begin{array}{l}
w(t, 0)=\frac{\partial w}{\partial x}(t, 0)=0 \\
\mathcal{M}_{\text {int }}(t, \ell)=\frac{\partial \mathcal{M}_{\text {int }}}{\partial x}(t, \ell)=0
\end{array}\right\} \quad, \quad t>0,
\end{aligned}
$$

along with appropriate initial conditions, as a model for characterizing the transverse beam dynamics. As detailed in [7], the composite density and internal bending moment are given by

$$
\begin{aligned}
& \rho(x)=\rho_{b} h b+2 \rho_{r} b_{r} \ell_{r} \chi_{r o d}(x) \\
& \mathcal{M}_{\text {int }}(t, x)=E I(x) \frac{\partial^{2} w}{\partial x^{2}}(t, x)+c_{D} I \frac{\partial^{3} w}{\partial x^{2} \partial t}(t, x)
\end{aligned}
$$

where the characteristic function $\chi_{\text {rod }}$ delineates the location of the rods and

$$
\begin{aligned}
& E I(x)=\frac{E_{b} h^{3} b}{12}+2 A_{\text {mag }} E^{H}\left(h / 2+\ell_{r}\right)^{2} \chi_{\text {rod }}(x) \\
& c_{D} I(x)=\frac{c_{D_{b}} h^{3} b}{12}+2 A_{\text {mag }} c_{D}^{H}\left(h / 2+\ell_{r}\right)^{2} \chi_{\text {rod }}(x) .
\end{aligned}
$$

To specify the external moments $\mathcal{M}_{\text {mag }}$, it is necessary to quantify the strains generated by an input field $H$ to the actuator. While the strains depend upon a variety of material prop- 


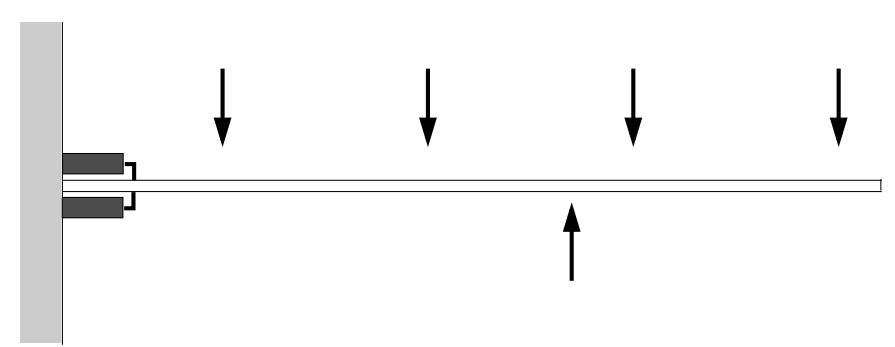

Figure 5. Cantilever beam with magnetostrictive actuators. Uniform force inputs are depicted above the beam while the measurement point is indicated by the lower arrow.

erties including the crystalline anisotropy, to first approximation they can be specified by the bulk magnetostriction

$$
\lambda(t)=\frac{3 \lambda_{s}}{2 M_{s}^{2}} M^{2}(t)
$$

where $\lambda_{s}$ denotes the saturation magnetostriction and $M$ is the magnetization given by (7). The combination of (7) and (12) provides a relation between the input field $H$ and positive strains generated by the actuator. To obtain bi-directional strains, the weighted magnetization $2 M(t) M_{s}$ is used to bias to the center of the magnetic range. This yields the strains

$$
e(t)=\frac{3 \lambda_{s}}{2 M_{s}^{2}}\left[M^{2}(t)+2 M(t) M_{s}\right]
$$

along with the expression

$$
\mathcal{M}_{\text {mag }}(t, x)=\mathcal{K}^{M}\left[M^{2}(t)+2 M(t) M_{s}\right] \chi_{\text {rod }}(x)
$$

for the moment produced by diametrically out-of-phase Terfenol-D rods. Here the constant $\mathcal{K}^{M}$ is given by $\left(3 \lambda_{s} / M_{s}^{2}\right) A_{m a g} E^{H}\left(h / 2+\ell_{r}\right)^{2}$.

To obtain a weak form of the model, we take the state to be the displacement $w$ in the state space $X=L^{2}(0, \ell)$ with the inner product

$$
\langle\phi, \psi\rangle_{X}=\int_{0}^{\ell} \rho \phi \psi d x
$$

The space of test functions is taken to be $V=H_{L}^{2}(0, \ell) \equiv\left\{\phi \in H^{2}(0, \ell) \mid \phi(0)=\phi^{\prime}(0)=0\right\}$ with the inner product

$$
\langle\phi, \psi\rangle_{V}=\int_{0}^{\ell} E I \phi^{\prime \prime} \psi^{\prime \prime} d x
$$

A weak form of the model is then given by

$$
\int_{0}^{\ell} \rho \ddot{w} \phi d x+\int_{0}^{\ell} \gamma \dot{w} \phi d x+\int_{0}^{\ell} \mathcal{M}_{i n t} \phi^{\prime \prime} d x=\int_{0}^{\ell} \mathcal{M}_{m a g} \phi^{\prime \prime} d x+\int_{0}^{\ell} g \phi d x
$$

for all $\phi \in V$. It is in this form that we develop the approximation method and formulate the control problem. 
To approximate the dynamics of (13) in a manner amenable to control formulation, we employ a Galerkin discretization in the spatial variable to obtain a semidiscrete ODE system in time. Specifically, the spatial basis is taken to be $\left\{\phi_{j}\right\}_{j=1}^{m+1}$ where $\phi_{j}(x)$ denotes the $j^{\text {th }}$ cubic $B$-spline modified to satisfy the fixed left boundary condition. Approximate solutions

$$
w^{m}(t, x)=\sum_{j=1}^{m+1} w_{j}(t) \phi_{j}(x)
$$

are then considered in the subpace $V^{m}=\operatorname{span}\left\{\phi_{j}\right\}$. To obtain a vector ODE system, the infinite dimensional system (13) is restricted to $V^{m}$ and posed in first-order form to yield

$$
\begin{aligned}
& \dot{y}(t)=A y(t)+B(u(t))+G(t) \\
& y(0)=y_{0} .
\end{aligned}
$$

The component system matrices have the form

$$
\begin{aligned}
& A=\left[\begin{array}{cc}
0 & I \\
\widetilde{Q}^{-1} K & \widetilde{Q}^{-1} C
\end{array}\right] \\
& B(u(t))=\left[M^{2}(u(t))+2 M(u(t)) M_{s}\right]\left[\begin{array}{c}
0 \\
\widetilde{Q}^{-1} \widetilde{B}
\end{array}\right] \\
& G(t)=\left[\begin{array}{c}
0 \\
\widetilde{Q}^{-1} \tilde{g}(t)
\end{array}\right]
\end{aligned}
$$

where $y(t)=\left[w_{1}(t), \cdots, w_{m+1}(t), \dot{w}_{1}(t), \cdots, \dot{w}_{m+1}(t)\right]$ and

$$
\begin{array}{ll}
{[\widetilde{Q}]_{i j}=\int_{0}^{\ell} \rho \phi_{i} \phi_{j} d x} & {[\widetilde{B}]_{i}=\mathcal{K}^{M} \int_{m a g} \phi_{i}^{\prime \prime} d x} \\
{[K]_{i j}=\int_{0}^{\ell} E I \phi_{i}^{\prime \prime} \phi_{j}^{\prime \prime} d x} & {[\tilde{g}(t)]_{i}=\int_{0}^{\ell} g(t, x) \phi_{i} d x} \\
{[C]_{i j}=\int_{0}^{\ell} c_{D} I \phi_{i}^{\prime \prime} \phi_{j}^{\prime \prime} d x .} &
\end{array}
$$

Note that $u(t)=H(t)$ denotes the control input to the system.

From (16), we note that the hysteresis input operator has the form

$$
N(H(t))=B(H(t))=\left[M^{2}(H(t))+2 M(H(t)) M_{s}\right] b
$$

where $b$ denotes the $2(m+1)$ vector

$$
b=\left[\begin{array}{c}
0 \\
Q^{-1} \widetilde{B}
\end{array}\right] .
$$

The corresponding inverse is

$$
N^{-1}(H(t))=M^{-1}\left(-M_{s}+\sqrt{M_{s}^{2}+H(t)}\right) b
$$


where $M^{-1}$ denotes the solution to the differential equation (10).

The specific form of the optimal control is dependent on the nature of the exogenous force $g$ in (11) or (13) and resulting disturbance term $G$ in (15). For the discussion which follows, we consider exogenous forces which are periodic with fundamental period $\tau$. Such forces arise when modeling systems driven by oscillating or rotating components.

We now consider two techniques for formulating a linear optimal control problem. In the first case, we simply linearize the operator $B(H)$ about a given field level. This technique is commonly employed when magnetostrictive transducers are employed at low drive levels with magnetic biases and is applicable only when the transducer is operated in the nearly linear range. The second technique is based upon the filtering of control inputs using the inverse compensator. This control method is applicable throughout the operational range of the transducer.

To specify the control input $u(t)=H(t)$ in the first case, we will linearize about the coercivity value $u_{c}$ at which $M\left(u_{c}\right)=0$. In this case, the approximate linear operator $\mathcal{B}$ is

$$
\mathcal{B}=2 M_{s} \frac{d M}{d u}\left(u_{c}\right) b
$$

where

$$
\frac{d M}{d u}=\widetilde{\delta}(1-c) \frac{M_{a n}-M_{i r r}}{k \delta-\alpha\left[M_{a n}-M_{i r r}\right.}+\frac{c M_{s}}{a}\left[\operatorname{csch}^{2}\left(\frac{H_{e f f}}{a}\right)+\left(\frac{a}{H_{e f f}}\right)^{2}\right]
$$

for the anhysteretic model (1).

We then consider the minimization of

$$
J(u)=\frac{1}{2} \int_{0}^{\tau}\left[y^{T}(t) Q y(t)+u^{T}(t) R u(t)\right] d t
$$

subject to

$$
\begin{aligned}
& \dot{y}(t)=A y(t)+\mathcal{B} u(t)+G(t) \\
& y(0)=y(\tau) .
\end{aligned}
$$

Here $Q$ and $R$ respectively denote the matrices used to weight the state and control input. Under typical stabilizability and detectability assumptions (e.g., see [8]), the optimal control for (20) is given by

$$
u(t)=-R^{-1} \mathcal{B}^{T}[\Pi y(t)-r(t)]
$$

where $\Pi$ solves the algebraic Riccati equation $A^{T} \Pi+\Pi A-\Pi \mathcal{B} R^{-1} \mathcal{B}^{T} \Pi+Q=0$. The perturbation variable $r(t) \in \mathbb{R}^{2(m+1)}$ is obtained through solution of the periodic perturbation system

$$
\begin{aligned}
\dot{r}(t) & =-\left[A-\mathcal{B} R^{-1} \mathcal{B}^{T} \Pi\right]^{T} r(t)=\Pi G(t) \\
r(0) & =r(\tau) .
\end{aligned}
$$

Note that in applications, (23) is typically integrated backward in time from $\tau$ or forward in time from $-\tau$.

As will be demonstrated in subsequent examples, the application of the control $u(t)$ specified by (22) into the original system (15) is effective at low input levels but fails drastically at high drive levels as a result of unincorporated time delays due to the hysteresis. 
A control based on inverse compensation is obtained by employing the input operator

$$
\mathcal{B}=b
$$

where $b$ is given by (18). This yields the resulting input $u_{p}$. To obtain the optimal control for the original system (15), the input $u_{p}$ is filtered through the inverse to obtain

$$
v(t)=M^{-1}\left(M_{s}+\sqrt{M_{s}^{2}+u_{p}(t)}\right) .
$$

We note that while this requires the online integration of (10) to obtain $M^{-1}$, the operation is significantly less expensive than the online integration of (23) to obtain $r(t)$ or the integration required to obtain state estimates $y_{c}(t)$ when full state information is unavailable.

\section{Example 2.}

To illustrate the performance and limitations of the two techniques, we consider a beam of length $\ell=.4573 \mathrm{~m}$, width $b=.0203 \mathrm{~m}$ and thickness $h=.0016 \mathrm{~m}$ with the material parameters for the beam and actuators summarized in Table 1. It should be noted that the beam parameters are consistent with typical values for aluminum laboratory beams while the Terfenol parameters are within the range obtained for model fits to an experimental transducer [4]. For this choice of beam parameters, the first two natural frequencies for the system occur at $6.1 \mathrm{~Hz}$ and $38.3 \mathrm{~Hz}$. To account for the effects of parameter discontinuities due the actuators and damping in the system, it was necessary to obtain these values through a fast Fourier transform (FFT) of time domain data resulting from a simulated impact to the beam (it is not possible to obtain analytic expressions through separation of variables). The driving frequency in the numerical examples was chosen close to but not exactly concurrent with these natural frequencies. For this example, the choice $m=12$ was sufficient for resolving the beam dynamics in the frequency range considered and the reported results were obtained with $m=16$.

A spatially uniform exogenous force

$$
g(t, x)=g_{0}[\sin (14 \pi t)+\sin (26 \pi t)]
$$

was applied throughout the time interval $[0,2.5]$ to simulate a periodic pressure field on the beam. The uncontrolled trajectories at the point $\bar{x}=3 \ell / 5$ for $g_{0}=1$ are plotted in Figures $6 \mathrm{~b}$ and $7 \mathrm{a}$ while the trajectories obtained with $g_{0}=100$ are plotted in Figures $6 \mathrm{~d}$ and $7 \mathrm{~b}$. Both cases exhibit a beat phenomenon due to the close proximity of the $7 \mathrm{~Hz}$ driving frequency with the $6.1 \mathrm{~Hz}$ natural frequency of the beam.

We first consider the performance of the control based on the linearized operator $\mathcal{B}$ defined in (19). The trajectories which result when the input $u$ given by (22) is fed back into the hysteretic system (15) at time $t=0.45$ are plotted in Figures $6 \mathrm{~b}$ and $6 \mathrm{~d}$. The relationship between the input magnetic field $H=u$ and the resulting magnetization $M$ for the two cases are given in Figures $6 \mathrm{a}$ and $6 \mathrm{c}$. The results for $g_{0}=1$ illustrate that the method is very effective at low drive levels where the linear model is accurate. At the high drive levels at which the actuators are advantageous, however, the input $B(u)$ introduces significant hysteresis which 
acts as a time delay to the system. The result is a loss in control authority which is sufficiently severe to produce controlled beam trajectories which are larger in magnitude at certain times than the uncontrolled trajectories.

The failure of the control based on the linearized system, when employed at high drive levels, motivates the consideration of a control based on inverse compensation. In this case, the operator $\mathcal{B}=b$ yields an input $u_{p}$ which is filtered by the inverse compensator (24) before input to the system. As illustrated in Section 3, the resulting input $u$ to the plant is then precisely the control $u_{p}$ specified in the absence of hysteresis and nonlinear dynamics. The controlled trajectories for $g_{0}=1$ and $g_{0}=100$ are respectively plotted in Figures $7 \mathrm{a}$ and $7 \mathrm{~b}$. In this case, there is no loss of control authority at high drive levels, thus illustrating that this approach provides a significant advantage over the control design based on the linearized system. Finally, a comparison with the examples in [9] illustrates that the performance of the controller based on inverse compensation is comparable to that obtained when the functional (20) is minimized subject to the original system (15) but at a fraction of the computational cost.

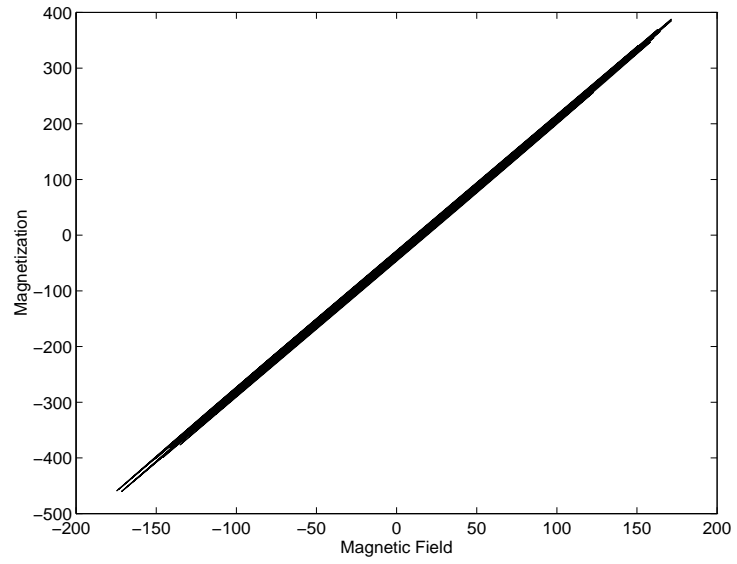

(a)

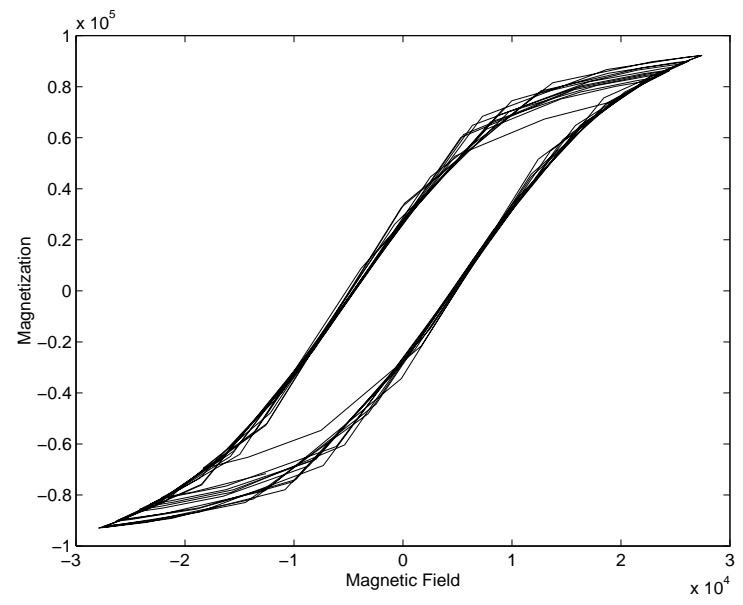

(c)

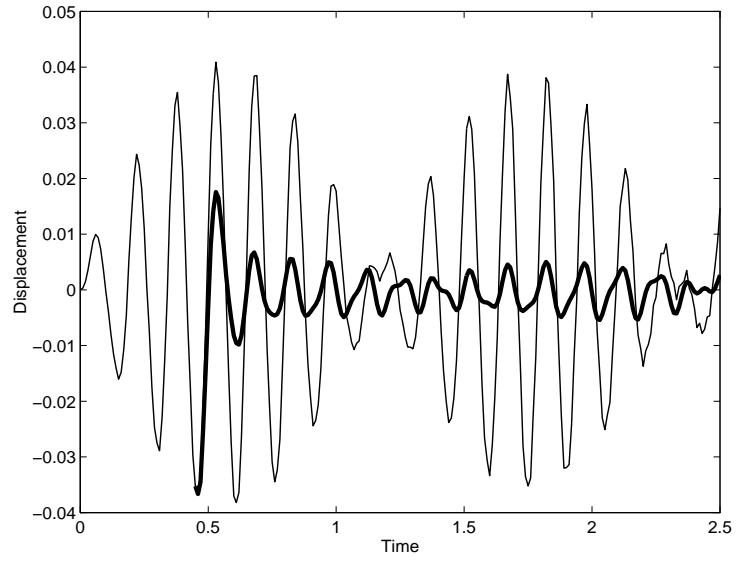

(b)

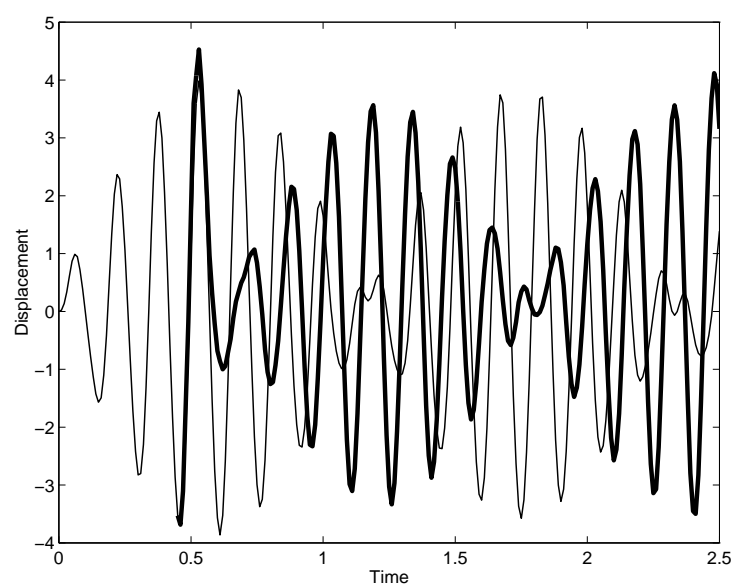

(d)

Figure 6. Feedback of the linear law (22) into the nonlinear system (15). Relationship between magnetic field $H$ and magnetization $M$; (a) $g_{0}=1$ and (c) $g_{0}=100$. Uncontrolled (—) and controlled (—) beam trajectories at $\bar{x}=3 \ell / 5 ;$ (b) $g_{0}=1$ and (d) $g_{0}=100$. 


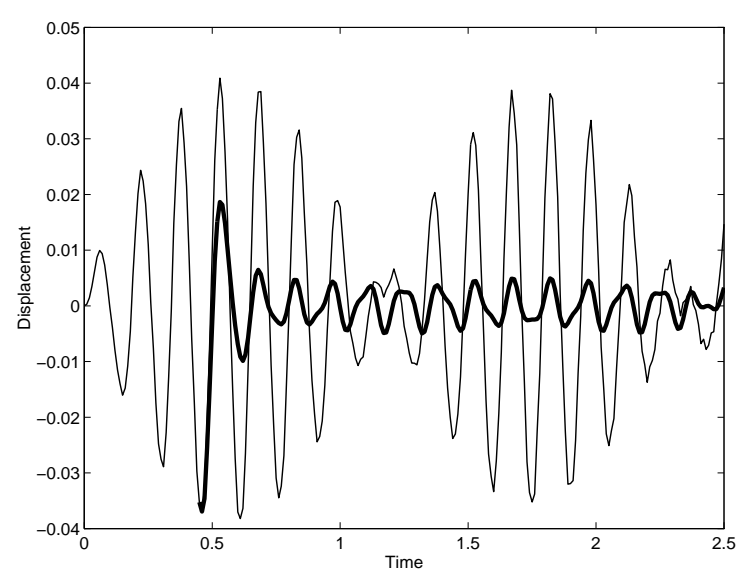

(a)

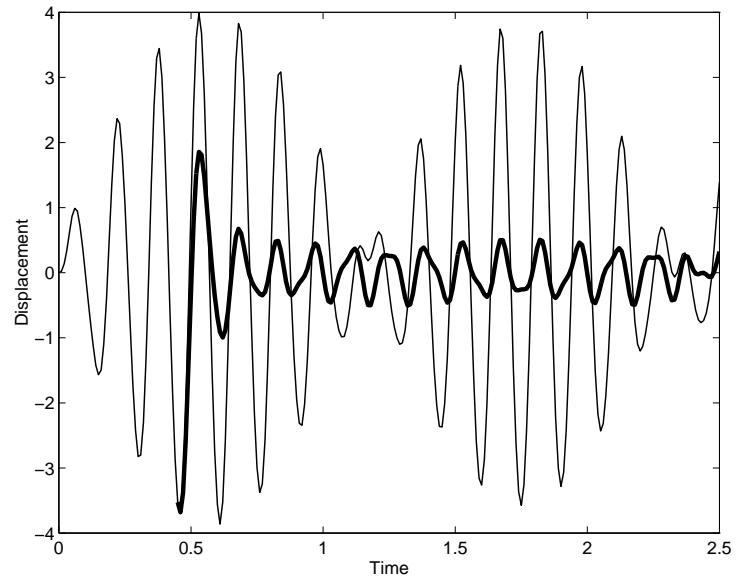

(b)

Figure 7. Uncontrolled beam trajectory (—) at the point $\bar{x}=3 \ell / 5$ and controlled trajectory (—) obtained with the filtered input (24); (a) $g_{0}=1$ and (b) $g_{0}=100$.

\begin{tabular}{l|l|l}
\hline Beam & Actuator & Terfenol \\
\hline$E_{b}=7.0861 \times 10^{10} \mathrm{~N} / \mathrm{m}^{2}$ & $E^{H}=7.0 \times 10^{10} \mathrm{~N} / \mathrm{m}^{2}$ & $a=7105 \mathrm{~A} / \mathrm{m}$ \\
$\rho_{b}=2863 \mathrm{~kg} / \mathrm{m}^{3}$ & $\rho_{r}=8524 \mathrm{~kg} / \mathrm{m}^{3}$ & $k=7002 \mathrm{~A} / \mathrm{m}$ \\
$c_{D_{b}}=9.3663 \times 10^{5} \mathrm{Ns} / \mathrm{m}^{2}$ & $\ell_{r}=.0254 \mathrm{~m}$ & $\alpha=.007781$ \\
$\gamma=.013 \mathrm{Ns} / \mathrm{m}^{2}$ & $b_{r}=.002 \mathrm{~m}$ & $c=0.3931$ \\
& $A_{\text {mag }}=.0064 \mathrm{~m}^{2}$ & $M_{s}=1.3236 \times 10^{5} \mathrm{~A} / \mathrm{m}$ \\
& & $\lambda_{s}=9.96 \times 10^{-4}$ \\
\hline
\end{tabular}

Table 1. Parameters for the beam and Terfenol transducer.

\section{Concluding Remarks}

This paper addresses the development of a modeling strategy and corresponding model-based inverse compensator for a class of ferromagnetic transducers including magnetostrictive actuators. Control designs based on linearized system models are typically ineffective in such regimes since they do not provide the capability for incorporating hysteresis and subsequent time delays. The result is a severe loss in control authority and potential destabilization of the system. To address this, we consider a model inverse which provides a transform so that linear inputs produce linear outputs when applied to the actuator.

The models are based on the Jiles-Atherton theory which quantifies the energy required to bend and translate domain walls pinned at inclusions in the materials. The construction of the models can be summarized in the following four steps: (1) Determine the effective field at a point in the material, (2) Quantify the anhysteretic magnetization through Boltzmann principles, (3) Quantify the irreversible magnetization due to domain wall translations and (4) Characterize the reversible magnetization due to domain wall bending. The total magnetization is then the sum of the irreversible and reversible components. 
The consolidated model quantifying the magnetization in ferromagnetic materials is in the form of an initial value problem which can be marched in time to characterize the hysteresis loop. A complementary differential equation then provides the inverse transforms for the actuators. The performance of this compensator and its employment in LQR control design are illustrated through numerical examples. It is noted that while the determination of the inverse requires the numerical approximation of a differential equation, the operation is significantly less expensive than the online integration required to compute perturbation variables or update state estimates. Hence the inverse compensator appears feasible for real-time experimental implementation.

Finally, we point out that while not discussed here, similar models have been developed to quantify hysteresis in certain ferroelectric materials [10]. Using techniques similar to those described here, the use of these models provides the capability for compensating for hysteresis observed in relaxor ferroelectric materials at low temperatures and piezoceramic actuators at high drive levels. The comprehensive development of inverse compensators based on ferroelectric domain wall theory is under investigation and will be reported in a future work.

\section{Acknowledgements}

The author would like to thank P.V. Kokotović, University of California, Santa Barbara, for input regarding the inverse compensation techniques. Sincere thanks are also extended to F.T. Calkins, The Boeing Company, and M. Dapino, A. Flatau and D. Jiles, Iowa State University, for extensive discussions regarding the construction, modeling and dynamics of magnetostrictive materials. This research was supported in part by the Air Force Office of Scientific Research under the grant AFOSR-F49620-98-1-0180.

\section{References}

[1] G. Tao and P.V. Kokotović, Adaptive Control of Systems with Actuator and Sensor Nonlinearities, John Wiley and Sons, New York, 1996.

[2] D.C. Jiles and D.L. Atherton, "Theory of ferromagnetic hysteresis," Journal of Magnetism and Magnetic Materials, 61, 1986, pp. 48-60.

[3] D.C. Jiles, J.B. Thoelke and M.K. Devine, "Numerical determination of hysteresis parameters for the modeling of magnetic properties using the theory of ferromagnetic hysteresis," IEEE Transactions on Magnetics, 28(1), pp. 27-35, 1992.

[4] F.T. Calkins, R.C. Smith and A.B. Flatau, "An energy-based hysteresis model for magnetostrictive transducers," ICASE Report 97-60; IEEE Trans. Magn., submitted.

[5] M.J. Dapino, R.C. Smith and A.B. Flatau, "An active and structural strain model for magnetostrictive transducers," Smart Structures and Materials 1998: Proceedings of the SPIE Conference on Smart Structures and Integrated Systems, San Diego, CA, March 1-5, 1998, pp. 198-209. 
[6] F.T. Calkins, R.L. Zrostlik and A.B. Flatau, "Terfenol-D vibration control of a rotating shaft," Proc. of the 1994 ASME International Mechanical Engineering Congress and Exposition, Chicago IL; In Adaptive Structures and Composite Materials Analysis and Applications AD-Vol. 45, 1996, pp. 267-274.

[7] R.C. Smith, "Modeling techniques for magnetostrictive actuators," CRSC Technical Report CRSC-TR97-6; Proceedings of the SPIE, Smart Structures and Materials 1997: Smart Structures and Integrated Systems, San Diego, CA, March 1997, Vol. 3041, pp. 243253.

[8] H.T. Banks, R.C. Smith and Y. Wang, Smart Material Structures: Modeling, Estimation and Control, Wiley/Masson, 1996.

[9] R.C. Smith, "A nonlinear physics-based optimal control method for magnetostrictive actuators," ICASE Report 98-4; Journal of Intelligent Material Systems and Structures, submitted.

[10] R.C. Smith and C.L. Hom, "A domain wall theory for ferroelectric hysteresis," 1999 SPIE Conference on Mathematics and Control in Smart Structures, submitted. 